\title{
LA PRÁCTICA EDUCATIVA...TRASUNTO DE LO HUMANO: FILOSOFAR LO HUMANO COMO TELEOLOGÍA EN LO PEDAGÓGICO-EDUCATIVO
}

Togliatty Toro

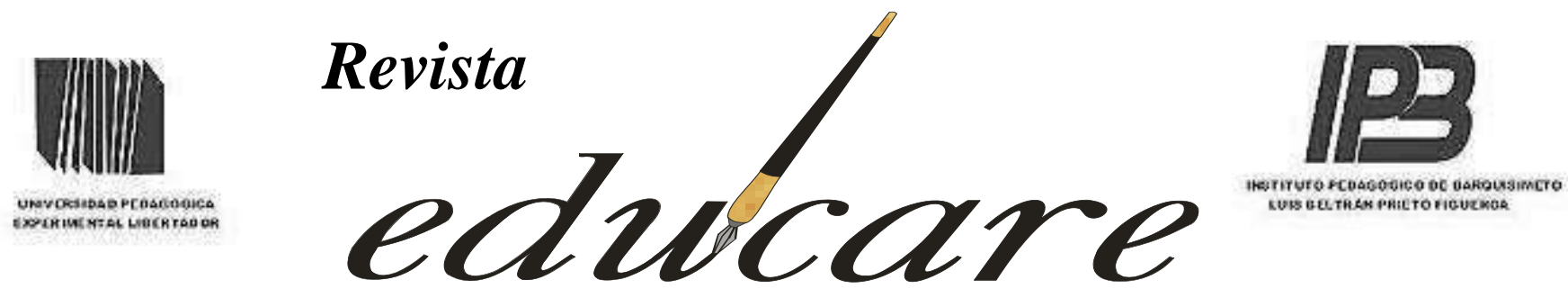

Órgano Divulgativo de la Subdirección de Investigación y Postgrado del Instituto Pedagógico de Barquisimeto "Luis Beltrán Prieto Figueroa”

Barquisimeto Estado Lara

Venezuela

\section{Volumen 23 № 3 Septiembre-Diciembre 2019}

\section{LA PRÁCTICA EDUCATIVA...TRASUNTO DE LO HUMANO: FILOSOFAR LO HUMANO COMO TELEOLOGÍA EN LO PEDAGÓGICO-EDUCATIVO}

THE EDUCATIONAL PRACTICE... THE HUMAN BACKGROUND: PHILOSOPHY THE HUMAN AS A PEDAGOGICAL-EDUCATIONAL TELEOLOGY

\section{S S N : $2244-7296$}

\author{
Autor \\ Togliatty Toro \\ Universidad Pedagógica Experimental Libertador
} (UPEL-IPB) 


\section{LA PRÁCTICA EDUCATIVA...TRASUNTO DE LO HUMANO: FILOSOFAR LO HUMANO COMO TELEOLOGÍA EN LO PEDAGÓGICO-EDUCATIVO \\ Togliatty Toro}

(pp.186-201)

Ensayo

Recibido: 23-06-2019

\section{RESUMEN}

La práctica educativa es la concreción de la educación, la pedagogía y el currículo. Como acción real de enseñanza la clase es el espacio del saber-cómo del docente. Analizar las implicaciones que en ella se llevan a cabo, en tanto momento de formación de la condición humana fue el objetivo, a los fines de mirar ésta como su teleología. Para la consecución de tal fin se seleccionó la tipología textual y se aplicaron las técnicas lectura evaluativa y presentación resumida de texto, a partir del fichaje documental. Los resultados revelan que la práctica educativa debe apuntalar hacia el proceso de hominización y un pedagogo reconoce que cuando enseña cualquier ciencia no refiere a la ciencia por la ciencia misma, sino ciencia para desarrollar al hombre y que cree optimistamente que la condición humana puede alcanzarse y en y por ello actúa consecuentemente y autorreflexiona de su hacer para educar.

Descriptores: práctica educativa; humano; pedagogía; educación; teleología
*Togliatty Toro

UPEL-IPB

Aceptado: 15-11-2019

\begin{abstract}
Educational practice is the realization of education, pedagogy and curriculum. As a real teaching action the class is the space of knowledge-how of the teacher. Analyzing the implications that are carried out in it, as long as the formation of the human condition was the objective, in order to look at this as its teleology. To achieve this purpose, the textual typology was selected and the techniques evaluative reading and summary presentation of text were applied, from the documentary signing. The results reveal that educational practice should propel the self-tyre process and a pedagogue recognizes that when he teaches any science he does not refer to science by science itself, but science to develop man and that he optimistically believes that the human condition can be attained and in and therefore acts accordingly and self-reflections of its doing to educate.
\end{abstract}

Key words: educational practice; human; pedagogy; education, teleology

\footnotetext{
* Profesora en Lengua Mención Literatura en la UPEL-IPB. Magíster en Literatura Latinoamericana y Caribeña. Doctora en Cultura Latinoamericana y Caribeña y Magíster en Gerencia Educacional. Investigadora profesional en: (a) Docencia de la Lengua y la Literatura; (b) Cultura, Lengua e Identidad; y, (c) Responsabilidad Social Universitaria, Currículo y Gestión Universitaria. Correo electrónico: togliattydejimenez@gmail.com
}

Volumen 23, $N^{\circ} 3$, Septiembre-Diciembre 2019. ISSN 2244-7296 


\section{LA PRÁCTICA EDUCATIVA...TRASUNTO DE LO HUMANO: FILOSOFAR LO HUMANO COMO TELEOLOGÍA EN LO PEDAGÓGICO-EDUCATIVO \\ Togliatty Toro}

(pp.186-201)

\section{Introducción}

Cuando Paulo Freire en 1970 afirmó que "Toda práctica educativa supone un concepto del hombre y del mundo" (p.56) nos dijo lo mismo que Fullat en 2000: educar es producir al ser humano. Ello porque el hombre es un animal que necesita educarse para ser.

Esto es -dixit Fullat-: un león o un elefante son educables para hacer lo que el hombre requiere que hagan. Pero ninguno deja de vivir en su propio mundo si esto no ocurriera. El hombre, por lo contrario, no pudiera vivir en su mundo si otro hombre no lo educara. Solo, no sería más que una bestia y su condición de hombre no pudiera desarrollarse.

He allí la medular valía de la educación como proceso de construcción de lo humano, en tanto el hombre no está prefabricado y ello produce que la educandidad sea una prerrogativa humana. En latín justamente se llamó dux o ducispor indicar el significado de guía o conductor; el hombre requiere un guía o conductor para educarse; de allí emergió el concepto educatio como relativo a la acción de educar al hombre (Fullat, ob. cit.).

La educación es, así, la única vía de hacerse hombre; es más podemos pensar que hacerse hombre es educar-se. En esa medida, los hombres a lo largo de los siglos han educado a sus nuevos individuos. Niños y niñas, adolescentes y jóvenes son amamantados por la sociedad que los recibe para hacer que estos nuevos hombres y mujeres reciban educación, a los fines de ser quienes esa sociedad necesita y requiere. Probablemente, esta idea nos permite pensar cómo la perpetuación de lo cultural va implícita en ese proceso de amamantamiento de nuevos seres.

Diariamente, recibimos (lengua, tradiciones, creencias, ideologías, práctica, entre otros) de algún otro quien nos educa informaciones, conocimientos, valores, reflexiones, orientaciones, entre otros. Y más allá de un alguien, ese otro está representado por un grupo de otros: un conglomerado (social) que educa. La sociedad educa; es la idea que refiero.

Volumen 23, N³, Septiembre-Diciembre 2019. ISSN 2244-7296 


\section{LA PRÁCTICA EDUCATIVA...TRASUNTO DE LO HUMANO: FILOSOFAR LO HUMANO COMO TELEOLOGÍA EN LO PEDAGÓGICO-EDUCATIVO \\ Togliatty Toro}

(pp.186-201)

Eso que recibimos de esa sociedad (y particularmente de cada otro con quien socializamos) ya se ha hecho cultura y los propios procesos sociales generan circulación. Por supuesto, hago esta afirmación sin negar que no podemos dejar de lado las posibilidades de resignificación cultural que cada sujeto, siendo dueño y protagonista de su propia vida, puede hacer al modernizar, cambiar e incluso transformar culturalmente lo que ha sido legado. En todo caso, lo central aquí es la discusión acerca de que cada sociedad, por ende, ha decidido antes que tales niños nacieran qué tipo de hombre deseaba forjar para sí misma y por ello estos fueron encaminados hacia tales o cuales vías de desarrollo del hombre: cada hombre pensado se educó en su sociedad tal y como ella así lo decidió.

Cuando refiero a la cultura probablemente dejo la idea de la educación no formal, esa inconsciente y no reflexiva del proceso de hacer hombre. Esta es real y efectiva en la vida de cada sujeto todos los días de su vida. Como ya aseveré, cada palabra, frase, norma, axioma, práctica social, consejo para hacer tal o cual cosa... trae consigo un aprendizaje que pone al sujeto cultural en la vía de ser sujeto en su determinada cultura. En esa medida es el contexto social el que "constituye al sujeto mismo y al contexto mismo" (Fornet-Betancourt, 2009) y lo que es el hombre y lo que somos cada uno de nosotros es la resulta del proceso de subjetivación. El hecho de ser no formal no hace demeritoria esta idea, en tanto lo humano se forma entre humanos como parte del proceso de intersubjetivación constante y diario.

Es más, fue esta educatio la única que recibió cada hombre y mujer desde la edad antigua hasta la edad media. El cambio social vino de la mano del Renacimiento como época histórica cuando emergió durante lo que hoy conocemos como sociedad moderna. Durante ella la educatio social del hogar y del contexto mismo dio paso a una educatio formal (de la enseñanza). Habermas (1980) al respecto afirmó que:

Lo que hoy entendemos por sociedad moderna adquirió forma durante el Siglo XVII en el rincón noroccidental del sistema europeo de sociedades, en Gran Bretaña, Holanda y Francia. El desarrollo subsiguiente de la sociedad moderna incluyó tres procesos de cambio estructural revolucionario: La

Volumen 23, $N^{\circ} 3$, Septiembre-Diciembre 2019. ISSN 2244-7296 


\section{LA PRÁCTICA EDUCATIVA...TRASUNTO DE LO HUMANO: FILOSOFAR LO HUMANO COMO TELEOLOGÍA EN LO PEDAGÓGICO-EDUCATIVO \\ Togliatty Toro}

(pp.186-201)

Revolución Industrial la Revolución Democrática y la Revolución Educativa (p. 49)

Nos interesa puntualmente centrarnos en esa revolución educativa. Fue el marco de la Modernidad como período cronológico lo que trastocó lo preexistente desde sus raíces, desde la concepción del hombre, pasando por las concepciones de las teorías del conocimiento, las artes, el tiempo, la conciencia, la sociedad, la ciencia y la moral, hasta alcanzar la concepción de Dios, la existencia y lo desconocido.

Ello ocurrió porque el elemento primordial de la Modernidad fue el hombre. Era sustancial abandonar el pensamiento mítico que lo había arrinconado hacia las esquinas de una gran casa (universo) que no veía -como los últimos hombres del Popol Vuh- y hacer que éste se mirara a sí mismo como el dueño de la razón, porque era su pensamiento (pensamiento crítico) lo que iba a permitirle "razonar" acerca del mundo que lo rodeaba.

Así, con la llegada del Renacimiento el hombre se vio a sí mismo y se auto-reconoció como eje del universo. Este cambio propugnó un "giro copernicano" que generó una revolución de fondo en la historia mundial, no solo porque el hombre moderno, rebelado ya, comenzó a transitar un camino sólo escrito por él, sin las ataduras de lo tradicional, lo dogmático y lo normativo, sino porque este nuevo hombre y su conciencia crítica permitió hacer demiurgos que pasaron de los estadios teológico y metafísico del conocimiento al estadio positivo -tal y como propuso Comte- (León Rugeles, 2012)

El hombre moderno produjo conocimiento positivo, el cual era un tipo de conocimiento que solo podía ser válido si era producto de lo verificado empíricamente, en el marco de unas representaciones lingüísticas de los hechos conocidas como teorías y desde la aplicación de un método centrado en la observación y la comprobación experimental conocido como el método científico.

Volumen 23, $N^{\circ} 3$, Septiembre-Diciembre 2019. ISSN 2244-7296 


\section{LA PRÁCTICA EDUCATIVA...TRASUNTO DE LO HUMANO: FILOSOFAR LO HUMANO COMO TELEOLOGÍA EN LO PEDAGÓGICO-EDUCATIVO \\ Togliatty Toro}

(pp.186-201)

La educatio del hombre viró radicalmente a las ciencias -creadas por el hombre mismo con el poder de su razón- para hacer de sí y de la sociedad lo que Habermas (1980) describió como una realidad de "ciencia moderna, el progreso infinito del conocimiento y el avance infinito hacia la mejoría social y moral” (p. 20). La conciencia modernista que se formó a raíz de este cambio modificó la condición humana. Lo que la sociedad privilegió en su hombre educado fue un tipo de conocimiento que ofrecía promesas: bienestar social, seguridad existencial de las identidades individuales (producto del ser dueño de sí mismo), una vida mejor y progresista (producto de los aportes al desarrollo que derivaba de las ciencias y el capitalismo). La sociedad privilegió el conocimiento científico.

Y si esa era el conocimiento (humano) que se privilegiaba, entonces fue preciso educar al hombre ya no en el hogar ni en la comunidad. Esos lugares sagrados de producción y circulación del conocimiento dieron paso a la escuela como un nuevo espacio sagrado donde el humano que la sociedad quería alcanzaría conocimiento científico.

La Modernidad inventó la escuela para la educatio moderna ¿Qué era ésta? Un lugar donde el conocimiento a aprender era todo un mundo fuera de la propia vida del hombre. Popper (citado en Flórez Ochoa, 2005) nominó este mundo como mundo tercero, refiriéndose a un mundo aparte de la realidad, cuya única existencia es resultado del producto humano: un mundo del conocimiento pleno de teorías, problemas científicos, discusiones científicas. Poseer ese mundo, apropiarse de él, ser sujeto de él formaba al humano como un nuevo tipo de hombre: un hombre profesional en alguna ciencia, de conocimiento científico, de escuela.

Me refiero aquí al humano como un hombre, cuya máxima era la de ser profesional en el marco de alguna ciencia, sapiente de su específico conocimiento científico del mundo tercero popperiano, formado metódicamente en la escuela. Enuncio el concepto escuela tal y como lo hizo Martín-Barbero en 2003: "entendiendo por escuela todo sistema educativo desde la primaria hasta la universidad" (p. 19), particularmente al considerar que este espacio

Volumen 23, $N^{\circ} 3$, Septiembre-Diciembre 2019. ISSN 2244-7296 


\section{LA PRÁCTICA EDUCATIVA...TRASUNTO DE LO HUMANO: FILOSOFAR LO HUMANO COMO TELEOLOGÍA EN LO PEDAGÓGICO-EDUCATIVO \\ Togliatty Toro}

(pp.186-201)

educativo se constituyó en un espacio de saber y de poder donde el saber otorgaba poder al hombre, quien era legitimado y validado por otras figuras sociales (maestros y compañeros de estudio).

La realidad moderna permitió así un nuevo escenario social, la escuela, como depositario de ese mundo tercero y con ello se propuso "una re-definición del sujeto de la educación" (Martín-Barbero, ob. cit.). La educatio -legitimada antes en el seno de las familias y comunidades- se movió hacia la escuela donde el humano (educable) se hacía hombre al modo del "sujeto cartesiano del conocimiento" (ob.cit., p. 17). Fue ésta la mayor mutación humana -dixit Barbero- y "la base de la actual acción educativa de la escuela actual" (ibídem) en la cual el hombre motorizaba un tipo de circulación del saber como fuente clave de poder: a más conocimiento científico, mayor poder.

Hubo otro hombre necesario: el sujeto guía o conductor de ese otro (humano) dentro de la escuela. El oficio de docente surgió -dixit Mora-García (2000)- porque un profesional distinto se requería para enseñar al hombre, un hombre intelectual, cuyo trabajo ya no era el manual -común para todos- sino el saber académico (científico) soportado en un poderoso instrumento de conocimiento del cual era dueño: el libro.

Este hombre con oficio de educatio requería, por supuesto, poseer mayor saber. Ese saber provenía de la ciencia en la que educaba al otro. Ocurrió entonces el cambio que Habermas propuso, la sociedad que educaba de manera informal y cotidiana dentro de las familias y comunidades:

1. Entregó a un hombre la acción de educar y le permitió ejercer ese oficio con los nuevos hombres de su sociedad (niños y adolescentes),

2. Creó la escuela como espacio en el cual se encofró la circulación del saber y se atrincheró el conocimiento (Martín-Barbero, 2003),

3. Inauguró una práctica educativa, cuya concepción de educatio de lo humano mutó hacia la formación de un profesional sapiente del mundo tercero popperiano.

Volumen 23, $N^{\circ} 3$, Septiembre-Diciembre 2019. ISSN 2244-7296 


\section{LA PRÁCTICA EDUCATIVA...TRASUNTO DE LO HUMANO: FILOSOFAR LO HUMANO COMO TELEOLOGÍA EN LO PEDAGÓGICO-EDUCATIVO \\ Togliatty Toro}

(pp.186-201)

4. El ser humano que se deseaba formar era uno con conocimiento científico para el progreso, avance y desarrollo social (Flórez Ochoa, 2005).

La mutación producida ameritaba, en consecuencia, el surgimiento de tres conceptos que debo revisar detenidamente: pedagogía, enseñanza y currículo. Toda práctica educativa -vista como la acción real in situ donde se lleva a cabo la educatio- es la resulta de esos tres conceptos y es una muestra del devenir histórico que he tratado anteriormente, razón por la cual amerito desvelar estos conceptos, pero además sus interrelaciones existentes.

La pedagogía -en el marco de las ciencias paridas en la Modernidad- se abrió paso como "una disciplina que estudia y propone estrategias para lograr la transición del niño del estado natural al estado humano, hasta su mayoría de edad como ser racional, autoconsciente y libre" (Flórez Ochoa, ob. cit., p. 13). Su especificidad vino dada por la necesidad de estudiar cómo aprende el hombre y cuáles estrategias serían más eficaces y efectivas para alcanzar dicho fin. Posner (1998) agregó a esta conceptualización la idea de integración de todas las ciencias; según él la pedagogía se hizo de los aportes de las ciencias que estudian al hombre (sociología, filosofía, psicología, antropología, lingüística) para amalgamarlas en un todo integral que deviniera en modelos pedagógicos que contemplan un concepto de hombre, un proceso de hominización, un tipo de experiencia educativa, un tipo de intercambio entre educador y educando y un conjunto de métodos y técnicas como modelo de acción educativa.

Ser educador implicó, por supuesto, poseer conocimiento pedagógico para poder llevar a cabo la enseñanza. Ésta era la actividad humana que permitía a los aprendices apropiarse subjetivamente del mundo de las ciencias (Flórez Ochoa, 2005). Ser educador era ser enseñante, más para serlo también había que formarse en lo pedagógico.

El oficio se convirtió entonces en un oficio complejo, porque su puesta en escena jaloneaba hacia dos polos: el uno, propio de las ciencias donde el conocimiento científico y su producción era asunto del enseñar; y el otro, propio de la condición humana donde era preciso reconocer que por encima de las ciencias se formaban hombres en un específico

Volumen 23, $N^{\circ} 3$, Septiembre-Diciembre 2019. ISSN 2244-7296 


\section{LA PRÁCTICA EDUCATIVA...TRASUNTO DE LO HUMANO: FILOSOFAR LO HUMANO COMO TELEOLOGÍA EN LO PEDAGÓGICO-EDUCATIVO \\ Togliatty Toro}

(pp.186-201)

proceso de hominización. Uno y otro jaloneaban hacia sí y el enseñante podía (y puede) perder de vista lo humano o lo científico.

Meritorio sería aquí reconocer que la búsqueda moderna del saber racional nació para hacer de la vida del hombre una donde la promesa de felicidad fue la oferta: el hombre con su infinita capacidad humana de aprender y crear podía construir ciencias y máquinas que mejoraran su vida. Lo sustancial fue crear conocimiento que entonces produjera desarrollo humano. La revolución educativa fue y sigue siendo siempre en pro de lo humano; cualquier cambio educativo siempre ha sido para favorecer la concepción de hombre que deseamos alcanzar.

Esa es la causa fundamental de la defensa, particularmente, de Flórez Ochoa (2005) de la naturaleza sociohumanística de la pedagogía. Siendo que lo pedagógico puede confundirse con un saber científico-técnico, aunque ella no es solo saber instrumental en sí mismo; es saber instrumental para y ese para es la enseñanza. Bien afirmó el autor que "no se puede enseñar bien sin pedagogía” (p. 174).

Hay diferencias sustanciales en el acto de enseñar. Cualquiera puede educarnos, tal y como vimos que antes de la escuela moderna la educatio se celebraba. No cualquiera puede enseñarnos. La enseñanza implica ser pedagogo y ello remite al hecho de reconocer, de manera intencional, cuál es el hombre que desea formarse y actuar en consecuencia. Por eso, los saberes pedagógicos -como saberes únicos de los maestros- envuelven un cúmulo de conocimientos acerca de la meta a seguir, el plan a transitar, los contenidos a enseñar, los métodos y técnicas a usar en el proceso, los problemas a enfrentar y la forma de solucionar dichos problemas (Flórez Ochoa, ob. cit.), pero por encima de todo eso está la clara conciencia de lo humano.

¿Qué significa esta aseveración? El pedagogo en su enseñanza -vista como acción real de educación de otro en un aula de clase para cumplir intencionalmente un plan- reconoce que más allá del contenido o la ciencia que enseña, más allá de los recursos, técnicas y métodos

Volumen 23, $N^{\circ} 3$, Septiembre-Diciembre 2019. ISSN 2244-7296 


\section{LA PRÁCTICA EDUCATIVA...TRASUNTO DE LO HUMANO: FILOSOFAR LO HUMANO COMO TELEOLOGÍA EN LO PEDAGÓGICO-EDUCATIVO \\ Togliatty Toro}

(pp.186-201)

que usa, más allá de los libros, teorías, axiomas que lo acompañan, más allá incluso del propio escenario escolar donde se encuentren su meta, su norte brillante hacia el cual ir es el hombre ¿Por qué? El pedagogo cree en lo humano.

Cada docente que funge en el oficio de educador, de enseñante, de pedagogo posee y defiende lo que Flórez Ochoa (2005) llamó optimismo pedagógico. Este concepto pone a este particular hombre en la creencia de que el hombre es educable y que al serlo puede ser siempre mejor persona, y que tal logro es posible porque él (el enseñante) tiene claridad en ese plan intencional y esa meta de la condición humana. Retorno aquí a la idea que inauguró este texto:

1. Toda práctica educativa supone un concepto del hombre y del mundo de Freire.

2. Educar es producir al ser humano de Fullat.

Un pedagogo reconoce que lo que él efectivamente hace cuando enseña cualquier ciencia no es la ciencia por la ciencia misma; enseña ciencia para desarrollar al hombre quien haciendo uso de ella mejorará su vida, se desarrollará y autodesarrollará y con ello impactará la sociedad misma a la cual regresará luego de la escuela. Es por eso que, la pedagogía va más allá de las ciencias haciendo uso de ellas. Y es por eso que, los pedagogos trabajan para el proceso de hominización y creen que sí es posible desde una acción intencional diseñar estrategias para potenciar el desarrollo de los individuos.

¿Qué favorece este hecho? La propia condición humana. Así como he dicho que el hombre amerita de educandidad (porque si no se educa solo sería una bestia), así también reconozco que la propia naturaleza humana (del humano como especie) actúa a su favor. Según Flórez Ochoa (2005):

1. El hombre es autónomo: y desde esa autonomía tiene poder de decisión para hacer de sí lo que él mismo desea. Como especie le fue dada la capacidad mental para ser libre, para autodeterminar su conducta, para decidir cuáles valores regirán su vida e incluso, si fuere

Volumen 23, $N^{\circ} 3$, Septiembre-Diciembre 2019. ISSN 2244-7296 


\section{LA PRÁCTICA EDUCATIVA...TRASUNTO DE LO HUMANO: FILOSOFAR LO HUMANO COMO TELEOLOGÍA EN LO PEDAGÓGICO-EDUCATIVO \\ Togliatty Toro}

(pp.186-201)

necesario, para autoconstruirse libre y conscientemente. Digo yo: esta condición humana puede potenciarse con la educación.

2. El hombre tiene capacidad mental para apropiar información: y entonces acumular, analizar, sintetizar y objetivar información para aprender constantemente e incluso para desaprender y seguir aprendiendo sin fin. Digo yo: esta condición humana puede potenciarse con la educación.

3. El hombre posee unicidad en su pluralidad: sea quien fuere el sujeto que aprende, de cualquier etnia, religión, estrato social, formación ideológica... todos los hombres por más distintos que sean, todos aprenden porque todos en esencias son humanos. Digo yo: esta condición humana puede potenciarse con la educación.

Entonces, debe quedar claro al pedagogo que tiene en sus manos una materia prima que coadyuva a su labor. La condición humana no solo es objeto de educandidad, sino que la favorece y beneficia. Incluso los hombres que han decidido dejar de serlo pueden resarcir el camino recorrido y retomar la senda perdida, porque solo el hombre (como especie) puede autoconstruirse y autopreservarse. La inteligencia que le fue dada a la especie humana le permite protegerse a sí misma, incluso de sí.

Claro que no podemos negar que en el marco de esa autonomía, de esa capacidad mental y esa unicidad haya hombres quienes decidan la deshumanización como alternativa de construcción. Así como la educandidad nos mostró la idea de la necesidad de educar al hombre, también nos muestra su inconclusión. Freire (1970) nos dijo que tanto humanización como deshumanización son "posibilidades de los hombres como seres inconclusos y conscientes de su inconclusión" (p. 40).

Pero esa posibilidad es solo cuestión del afuera de la escuela. Si hablamos de la escuela, como espacio de lo pedagógico, la deshumanización es solo una distorsión del ser. Los hombres en la escuela se humanizan entre sí, porque la razón de ser de ella es justamente formar al hombre, educarlo para hacer de él el hombre.

Volumen 23, $N^{\circ} 3$, Septiembre-Diciembre 2019. ISSN 2244-7296 


\section{LA PRÁCTICA EDUCATIVA...TRASUNTO DE LO HUMANO: FILOSOFAR LO HUMANO COMO TELEOLOGÍA EN LO PEDAGÓGICO-EDUCATIVO \\ Togliatty Toro}

(pp.186-201)

¿Cómo lo educa para lograrlo? La respuesta remite al último concepto por dilucidar: el currículo. Cuando la educatio era responsabilidad de la familia y la comunidad había una concepción del hombre a formar, pero no había currículo. Cuando la educatio fue asumida por la escuela _y entonces por el pedagogo, educador, enseñante_ hubo que predeterminar el hombre que deseaba formar. Por supuesto que esta decisión del hombre que deseamos en una sociedad es siempre decisión de esa sociedad. Los contratos sociales y políticos (como la Constitución del país) así lo establecen, pero para alcanzar tal concepción de hombre se establece un plan, un camino que el hombre debe seguir para.

La escuela es un espacio de aprendizaje donde se camina un camino ya trazado. Es a eso lo que llamamos currículo. La pedagogía ha proporcionado un cúmulo de teorías acerca de qué y cómo hacer para enseñar al hombre. Pues, el currículo hace concreta esa teoría pedagógica para formar a un tipo de hombre profesional particular. Posner (1998) al respecto manifestó que "un currículo es un plan de construcción (y formación) que se inspira en conceptos articulados y sistemáticos de la pedagogía...que pueden ejecutarse en un proceso efectivo y real llamado enseñanza" (p. xvii).

Encontramos en tal definición dos elementos que permiten distinguir lo curricular: uno que refiere al plan o trayecto formativo del hombre; plan que está predeterminado con antelación, que es intencional y que cumple alguna teoría pedagógica; y otro que refiere al hecho de que se hace concreción en un hecho de enseñanza real. Ambos elementos nos muestran que lo curricular contiene la decisión de hacer a un tipo de hombre y a la acción misma en sí.

Cada clase (de cada día) es currículo, pero ésta es efectivamente el cumplimiento real de una teoría pedagógica, un paso entre todo un camino y una acción donde formamos al hombre que queremos. Una clase es, en esencia, la educatio (formal) en pequeño; una clase en enseñanza real de un pedagogo; una clase es pedagogía en acción; una clase es microcurrículo.

Volumen 23, $N^{\circ} 3$, Septiembre-Diciembre 2019. ISSN 2244-7296 


\section{LA PRÁCTICA EDUCATIVA...TRASUNTO DE LO HUMANO: FILOSOFAR LO HUMANO COMO TELEOLOGÍA EN LO PEDAGÓGICO-EDUCATIVO \\ Togliatty Toro}

(pp.186-201)

Para llegar a tal acción educativa es meritorio que quien hace de guía y conductor reconozca clara y conscientemente lo que es una clase, lo que es su clase. Sin esa mirada amplia de su acción docente, difícilmente la práctica educativa cumple su cometido. Afirmo, entonces, que una clase encarna la educación, la pedagogía, la enseñanza y lo curricular para la formación humana.

Pensar una clase, idearla, planearla... parecía ser solo ámbito de lo técnico-instrumental, era solo acción técnica, cuya intención era la producción del conocimiento práctico que lo pedagógico-curricular ameritaba. Carr en 2002 explicó que la práctica educativa no es una tarea solo regida por la teoría (pedagógica); si bien es cierto la acción real de enseñar es una concreción curricular donde aplicamos una teoría pedagógica, ello no significa que en el decurso de la acción la misma situación práctica en la que se aplica esa teoría no emerjan condiciones y necesidades qué evaluar.

Ello nos trae a un último concepto: praxis educativa. La práctica educativa es el hecho real donde se ejecuta lo pedagógico y lo curricular, en el marco de un contexto particular (social, cultural y escolar) y un escenario específico de aula. Todo pedagogo obtiene de su acción real saberes producto de la práctica. Durante ella, Carr afirmó que se aprende desde phronesis: juicio sabio de acción. El pedagogo aprende en la práctica, sabiamente, a actuar en función de la teoría pedagógica que debe desarrollar, de acuerdo al currículum que fue establecido.

Esto incide en su acción, porque ésta es acción educativa que activa la teoría pedagógica en la manera que el contexto y el escenario lo permiten; y, de la misma manera, aprende a hacer las adecuaciones que sean necesarias y a las que hubiere lugar en su caso concreto.

¿Qué implicaciones tiene esto en la práctica educativa? El mismo Carr nos mostró: como resulta de la phronesis la teoría no se queda solo en ella, sino que la acción reflexiva del docente puede modificar la teoría, enriquecerla, mejorarla para los casos concretos donde se actúa. Un pedagogo tiene un saber-cómo para la práctica educativa, pero es en la misma

Volumen 23, $N^{\circ} 3$, Septiembre-Diciembre 2019. ISSN 2244-7296 


\section{LA PRÁCTICA EDUCATIVA...TRASUNTO DE LO HUMANO: FILOSOFAR LO HUMANO COMO TELEOLOGÍA EN LO PEDAGÓGICO-EDUCATIVO \\ Togliatty Toro}

(pp.186-201)

práctica que él -como expresión de un humano que es autónomo, libre, consciente, autoconstructor de sí, inteligente, reflexivo- puede discernir entre buenas y malas prácticas educativas, a la vez que puede reconocer qué está mal y bien, qué y cómo debe mejorar o cambiar, qué puede producir como adecuaciones pedagógicas efectivas para alcanzar lo que curricularmente fue establecido como meta.

El saber-cómo da paso al saber-reflexivo. Pero sin práctica educativa no hay praxis educativa. Debe haber acción real educativa para que en el seno de ésta emerja la acción reflexiva y consciente del docente quien reconoce su práctica y camina hacia hacerla mejor, pedagógica y curricularmente.

Práctica educativa y praxis educativa son dos polos preeminentes de la acción real de enseñar. Claro que no toda práctica educativa genera praxis educativa. Hoy, la teoría pedagógica crítica (ob. cit.) promueve la necesidad de formar al docente en autorreflexión de sí para que su práctica educativa sea constantemente objeto de mirada evaluadora y de mejora educativa.

¿Qué quisiéramos evaluar? ¿Qué tipo de autorreflexión docente quiere promoverse? El criterio de validación de la educatio que Flórez Ochoa defiende incesantemente es el pedagógico $\mathrm{y}$, en esa medida, sostiene que una práctica educativa es pedagógica cuando logra el desarrollo de la condición humana.

Por eso retorno a la concepción del propio autor que ya referí: la pedagogía es una disciplina cuya esencia está ligada al estudio de la transición del estado natural del hombre al estado humano ¿Cuál?: la bestia no educada que es al nacer al sujeto educable en su humanidad esencial que no es más que la de ser un hombre racional, autoconsciente y libre.

Racional para pensar, criticar, juzgar, valorar, reflexionar de sí y del mundo que lo rodea lo cual le permite incluso cambiar y transformar su realidad. Autoconsciente de su vida y su derredor, en una proporción que le permite reconocer que él tiene el poder de decisión moral y ético para hacer de sí y de ese derredor lo que desee y también para valorar su devenir

Volumen 23, $N^{\circ} 3$, Septiembre-Diciembre 2019. ISSN 2244-7296 


\section{LA PRÁCTICA EDUCATIVA...TRASUNTO DE LO HUMANO: FILOSOFAR LO HUMANO COMO TELEOLOGÍA EN LO PEDAGÓGICO-EDUCATIVO \\ Togliatty Toro}

(pp.186-201)

histórico y cambiarlo si fuere necesario. Libre para ser lo que haya decidido ser, incluso libre de no humanizarse si fuera esa su acción consciente; aunque la libertad que la escuela privilegia es la libertad de acción consciente para mejorar constantemente como persona, a lo cual coadyuva su racionalidad.

La práctica educativa debe apuntalar hacia estas búsquedas. La praxis educativa debe tenerlas presentes por si acaso en alguna manera se pierde del rumbo hacia lo humano. Lo mejor de esto es que, siempre los hombres entre sí tienen la capacidad de cambiar y transformar lo que no está bien; siempre es posible volver atrás y encontrar el camino; siempre es posible enmendar lo mal hecho. La propia condición humana nos impulsa a creer en ello y la propia humanidad nos apaña en la decisión de cambiar lo que pedagógica y curricularmente esté hecho anti-pedagógicamente.

Lo único que no admitimos es no instar una y otra vez a que entre todos nos humanicemos: para ser mejores personas, constantemente. Porque en definitivas cuentas, “el hombre no termina de serlo; antes bien es un enriquecimiento, un despliegue libre y expresivo de la propia espiritualidad que se va forjando desde el interior en el cultivo de la razón y de la sensibilidad" (Flórez Ochoa, 2005, p. 108).

Lo humano, en consecuencia, no termina, no es finito. Es un constante crecer de lo racional y lo sensible. Pero no estriba su esencia ni en objetivos, ni en contenidos, sino en lo que se hace del ser humano. En el ser mismo que se forja como resulta de ese constante proceso formador... "es lo que queda, es el fin perdurable" (ob. cit., p. 109).

Sea como sea, los hombres en la sociedad siguen educando a los otros y educándo-se entre sí, probablemente humanizándose o deshumanizándose. Pero dentro de la escuela, el único camino es la práctica educativa para la humanización. Esa es nuestra teleología diaria y sempiterna.

Volumen 23, $N^{\circ} 3$, Septiembre-Diciembre 2019. ISSN 2244-7296 


\section{LA PRÁCTICA EDUCATIVA...TRASUNTO DE LO HUMANO: FILOSOFAR LO HUMANO COMO TELEOLOGÍA EN LO PEDAGÓGICO-EDUCATIVO \\ Togliatty Toro}

(pp.186-201)

\section{Referencias}

Carr, W. (2002). Una teoría para la educación: hacia una investigación educativa crítica. ( $3^{\circ}$ Ed.) Madrid: Morata.

Flórez Ochoa, R. (2005). Pedagogía del conocimiento. Madrid: McGraw Hill

Fornet-Betancourt, R. (2009). Interculturalidad en procesos de subjetivización. México. D.F: Coordinación General de Educación Intercultural y Bilingüe.

Freire, P. (1970-2005). Pedagogía del oprimido. México: Siglo XXI

Fullat, O. (2000). Filosofías de la educación: Paideia. Barcelona: Ceac

Habermas, J. (1980). La modernidad un proyecto incompleto. En Foster, H. (1986). La posmodernidad. ( $7^{\circ}$ Ed.) Barcelona: Kairós

León Rugeles, F. (2012). Teoría del conocimiento. ( $3^{\circ}$ Ed.) Valencia: Universidad de Carabobo

Martín-Barbero, J. (2003). Saberes hoy: diseminaciones, competencias y transversalidades. Revista Iberoamericana de Educación. $\mathrm{N}^{\circ} 32$. pp. 17-34.

Mora-García, J. P. (2000). Universidad, currículum y postmodernidad crítica. Cuadernos del Doctorado. Barquisimeto: Doctorado Interinstitucional en Educación UCLA-UNEXPOUPEL.

Posner, G. (1998). Análisis de currículo. (2 Ed.) Santafé de Bogotá: McGraw Hill

Volumen 23, $N^{\circ} 3$, Septiembre-Diciembre 2019. ISSN 2244-7296 